\title{
MicroRNA-1246 Suppresses the Metastasis of Breast Cancer Cells by Targeting the DRAK1A/PGRN Axis to Prevent the Epithelial-Mesenchymal Transition
}

\author{
Pan Wang \\ Taizhou University \\ Wenju Chen \\ Taizhou University \\ Yaqiong Zhang \\ Taizhou University \\ Qianyi Zhong \\ Taizhou University \\ Zhaoyun Li \\ Taizhou University \\ Yichao Wang ( $\nabla$ tzyyycw@sina.com) \\ Taizhou University https://orcid.org/0000-0002-3456-0765
}

\section{Research}

Keywords: MicroRNA-1246, Breast cancer, Epithelial-mesenchymal transition, Dual-specificity tyro-sine(Y)-phosphorylation-regulated kinase 1A, Progranulin

Posted Date: May 17th, 2021

DOI: https://doi.org/10.21203/rs.3.rs-516130/v1

License: (c) (i) This work is licensed under a Creative Commons Attribution 4.0 International License. Read Full License 


\section{Abstract}

Objective. Breast cancer is one of the most common malignant and highly heterogeneous tumors in women. MicroRNAs (miRNAs), such as miR-1246, play important roles in various types of malignant cancers, including triple-negative breast cancer (TNBC). However, the biological role of miR-1246 in TNBC has not yet been fully elucidated. In this study, we studied the role of miR-1246 in the occurrence and development of TNBC and its mechanism of action.

Methods. Cell Counting Kit-8 (CCK-8), wound healing, and Transwell assays were performed to observe the effects of miR-1246 on TNBC cell proliferation, migration, and invasion, respectively. The expression of epithelial-mesenchymal transition (EMT) markers was detected by western blotting. Dual luciferase reporter assays were performed to determine whether DYRK1A is a novel target of miR-1246. In addition, an immunoprecipitation experiment was performed to verify the binding of DYRK1A to PGRN. Rescue experiments were performed to determine whether DYRK1A is a novel target of miR-1246 and whether miR-1246 suppresses the metastasis of breast cancer cells by targeting the DRAK1A/PGRN axis to prevent the epithelial-mesenchymal transition.

Results. Our results show that miR-1246 suppresses the proliferation, migration, and invasion of TNBC cells and that DYRK1A is a novel target of miR-1246. MiR-1246 plays a suppressive role in the regulation of the EMT of TNBC cells by targeting DYRK1A. DYRK1A mediates the metastasis of triple-negative breast cancer via activation of the EMT. We identified PGRN as a novel DYRK1A-interacting protein. DYRK1A and PGRN act together to regulate the occurrence and development of breast cancer through miR-1246.

Conclusion. miR-1246 attenuates TNBC cell invasion and the EMT by targeting the DRAK1A/PGRN axis. Our data suggest that miR-1246 may be used to develop novel early-stage diagnostic and therapeutic strategies for TNBC.

\section{Introduction}

Breast cancer is a common malignant tumor treated in clinical practice. According to the 2020 National Cancer Report issued by multiple national cancer centers, breast cancer ranks first in terms of incidence among women in the world (1). Triple-negative breast cancer (TNBC) refers to a subtype of breast cancer in which the estrogen receptor, progesterone receptor and human epidermal growth factor receptor are not expressed. It presents with unique molecular characteristics and biological behavior. Because of its late diagnosis, early recurrence, strong invasiveness, and high metastasis, triple-negative breast cancer is associated with a high mortality rate (2). Therefore, it is necessary and particularly urgent to explore the mechanism of invasion and metastasis of triple-negative breast cancer and explore the corresponding intervention measures.

TNBC is a malignant tumor derived from breast epithelial cells, and the epithelial-mesenchymal transition (EMT) can enable tumor cells derived from epithelial cells to acquire a highly invasive and metastatic 
phenotype, leading to tumor invasion, invasion and metastasis (3). The EMT is mainly manifested as the cellular loss of epithelial characteristics, such as decreased expression of E-cadherin on the cell surface, and acquisition of mesenchymal cell characteristics, such as increased expression of mesenchymal phenotypes, including vimentin and $\mathrm{N}$-cadherin, and high migratory ability. This process usually occurs during embryonic development. However, after cells undergo malignant transformation, transformed malignant cells undergo EMT. The tight junctions of epithelial cells undergoing the EMT dissociate, and cell adhesion is lost, leading to increased cell invasion and metastasis $(4,5)$. Therefore, the EMT is currently considered to be the first and most important step in the occurrence of malignant tumor invasion and metastasis. Many studies have shown that microRNAs can play roles in the EMT process by participating in the regulation of signaling pathways or transcriptional regulators $(6,7)$.

MicroRNAs (miRNAs) constitute a large class of small noncoding RNAs with a length of approximately 18-25 bp that are widely present in organisms and can posttranscriptionally modulate target gene expression by binding to the 3-'UTR of target gene mRNA (8). MiRNA regulation thus participates in the physiological and pathological processes of cells. Studies have shown that miRNA, as a small RNA that regulates posttranscriptional gene expression, is closely related to the EMT of cells in tumors (6). Among these miRNAs, miRNA-1246 family members are abnormally expressed in a variety of tumors, indicating that they play important roles in tumorigenesis and development (9), but the specific mechanism of miRNA-1246 in mediating triple-negative breast cancer is still unclear.

In this study, we explored the functional role of miR-1246 in triple-negative breast cancer cells, confirming that dual-specificity tyro-sine-(Y)-phosphorylation-regulated kinase 1A (DYRK1A) is a direct target of miR1246 and that miR-1246 suppresses the metastasis of breast cancer cells by preventing the epithelialmesenchymal transition through its effect on the DRAK1A/PGRN axis. The findings suggest that miR1246 may be a potential therapeutic target for the treatment of TNBC.

\section{Materials And Methods}

Cell lines and transfection. MDA-MB-231 and BT-549 cells were obtained from the Chinese Academy of Sciences (Shanghai, China). The MDA-MB-231 cells were cultured in Dulbecco's modified Eagle's medium (DMEM; Thermo Fisher Scientific, Inc., Waltham, MA, USA), and the BT-549 cells were cultured in RPMI1640 medium (Sigma-Aldrich, St. Louis, MO, USA); both media were supplemented with $10 \%$ fetal bovine serum (FBS; Thermo Fisher Scientific, Inc.), $100 \mathrm{IU} / \mathrm{ml}$ penicillin and $100 \mathrm{IU} / \mathrm{ml}$ streptomycin (both from Thermo Fisher Scientific, Inc.) at $37^{\circ} \mathrm{C}$ in a humidified atmosphere containing $5 \% \mathrm{CO}_{2}$. The appropriate number of cells was seeded into 6-well plates, and cell growth confluence reached approximately $80 \%$ before transfection was performed with Lipofectamine 3000 (Invitrogen, L3000015) transfection reagent, according to the manufacturer's instructions.

Reverse transcription-quantitative polymerase chain reaction ( $R T$-qPCR) assay. Total RNA was extracted from the cells using TRIzol reagent (Invitrogen; Thermo Fisher Scientific, Inc.). For the detection of miR-1246 expression, a miRNA reverse transcription kit (Thermo Fisher Scientific, Inc.) was used to 
convert RNA into cDNA according to the manufacturer's instructions. qPCR was performed using a miRNA Q-PCR detection kit (GeneCopoeia, Rockville, MD, USA) on an Applied Biosystems 7500 thermocycler (Applied Biosystems; Thermo Fisher Scientific, Inc.). The final reaction volume was $20 \mu \mathrm{l}$, which included $1 \mu \mathrm{l}$ of cDNA, $10 \mu \mathrm{l}$ of PCR master mix (GeneCopoeia), $2 \mu \mathrm{l}$ of primer and $7 \mu \mathrm{l}$ of $\mathrm{H}_{2} \mathrm{O}$. The PCR conditions were as follows: $95^{\circ} \mathrm{C}$ for 5 min and 40 cycles of denaturation at $95^{\circ} \mathrm{C}$ for $15 \mathrm{sec}$ and annealing/elongation at $60^{\circ} \mathrm{C}$ for $30 \mathrm{sec}$. Primers with the following sequences were purchased from Sangon Biotech Co., Ltd. (Shanghai, China): miR-1246 forward, 5'- TGAAGTAGGACTGGGCAGAGA -3' and reverse, 5'- TTTGGGTCAGGTGTCCACTC -3'; and U6 forward, 5'-CGCTTCGGCAGCACATATAC-3' and reverse, 5'-AAATATGGAACGCTTCACGA-3'. The U6 gene was used as an internal reference. The relative expression levels were analyzed using the $2-\Delta \triangle \mathrm{Cq}$ method, which was described in our previous publication (10).

Immunoprecipitation and immunoblotting. Cells were lysed with RIPA lysis buffer and extraction buffer. Then, the samples were precleared by incubation with $20 \mu \mathrm{l}$ of protein $\mathrm{G}$ beads (Amer-sham Biosciences) for 2 hours, followed by incubation overnight with 1-2 $\mu \mathrm{g}$ of the anti-PGRN primary antibody (Abcam, ab108608) at $4^{\circ} \mathrm{C}$. Protein $\mathrm{G}$ beads were then added to the sample tube and further incubated for 2 hours. The samples were then centrifuged at $10,000 \mathrm{~g}$ for $1 \mathrm{~min}$ at $4^{\circ} \mathrm{C}$, and the pellets were washed three times with immunoprecipitation buffer. For immunoblot analysis, bound proteins were boiled at $100^{\circ} \mathrm{C}$ for 10 min in SDS-PAGE loading buffer, and then, a western blot assay was performed.

Western blot analysis. Total protein was extracted according to the manufacturer's instructions of the protein extraction kit, and protein concentrations were measured via the BCA method. The proteins were subjected to $10 \%$ SDS-PAGE for electrophoresis and then transferred to a PVDF membrane. The membrane was blocked with 5\% skim milk at room temperature for 2 hours and then washed with TBST. The membrane was incubated overnight at $4^{\circ} \mathrm{C}$ with primary antibodies. The primary antibodies added included anti-DYRK1A (CST, 2271S), anti-N-cadherin (Abcam, ab124397), anti-E-cadherin (ICL, YT1454),

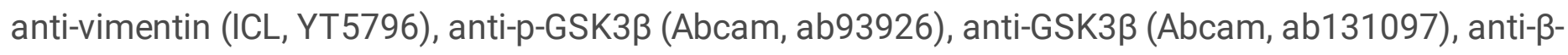
catenin (Proteintech, 51067-2-AP), anti-PGRN (Abcam, ab108608), anti-p-GSK3 $\beta$, anti-GAPDH (Goodhere Biological Technology, AB-P-R 001), and anti-lamin B (Boster, BA1228). After washing, the membranes were subsequently incubated with secondary antibody for 1 hour at $37^{\circ} \mathrm{C}$. An ECL chemiluminescence kit was used to visualize the protein bands. GAPDH was used as the loading control as described in our previous publication (10).

Cell proliferation assay. We used a CCK-8 kit (Dojindo, Kumamoto, Japan) according to the manufacturer's instructions to examine cell proliferation. Approximately $1 \times 10^{4} \mathrm{TNBC}$ cells were seeded into 96 -well culture plates. After 24,48 , or 72 hours of transfection, the cells were inoculated with $100 \mu \mathrm{L}$ of WST-8 at $37^{\circ} \mathrm{C}$ for 4 hours. The absorbance of the cells was then measured at OD 450 .

Cell migration assay. TNBC cells were cultured in a 6-well plate at a density of $1 \times 10^{5}$ cells per well. After 24 hours in culture, the cells were transfected with Lipofectamine 3000. After 12 hours, wounds (1-mm 
wide) were created using a plastic scriber. The scratch wounds were photographed over 24- and 48-hour periods using a microscope (CX31; Olympus Corporation, Tokyo, Japan).

Transwell assay. According to the manufacturer's protocol, Transwell experiments were performed to evaluate cell migration and invasion abilities. Briefly, $1 \times 10^{5}$ TNBC cells were prepared in serum-free DMEM or RPMI-1640 medium and added to the upper chamber of a Transwell plate coated with Matrigel (BD Biosciences, $\mathrm{NJ}$ ). Then, $500 \mu \mathrm{L}$ of DMEM or RPMI-1640 medium with $10 \% \mathrm{FBS}$ was added to the lower chamber containing MDA-MB-231 or BT-549 cells. After incubation for 24 hours, the cells were fixed using paraformaldehyde, stained with $0.1 \%$ crystal violet, and counted under a microscope (CX31; Olympus Corporation, Tokyo, Japan).

Luciferase reporter gene assay. Total cDNA from MDA-MB-231 cells was used to amplify the 3'UTR of DYRK1A using PCR, and the sequence was then cloned into a pmirGLO-Report vector (Thermo Fisher Scientific, Inc.), resulting in the generation of pmirGLO-DYRK1A. Mutations were introduced within the seed sequences of the 3'UTR of DYRK1A using a QuikChange site-directed mutagenesis kit (Stratagene; Agilent Technologies, Inc., Santa Clara, CA, USA). The mutated DYRK1A 3'UTR was then cloned into the pmirGLO-Report vector, generating pmirGLO-DYRK1A-Mut. 293T cells were cotransfected with $100 \mathrm{ng}$ of the pmirGLO -DYRK1A or pmirGLO-DYRK1A-Mut vector and $100 \mathrm{mM}$ miR-1246 mimic or scramble miR mimic, and a pRL-TK plasmid (Promega Corporation, Madison, WI, USA) was used for internal normalization. Following a 36 -hour incubation at $37^{\circ} \mathrm{C}$ in an atmosphere containing $5 \% \mathrm{CO}_{2}$, the cells were lysed using lysis buffer (Promega Corporation), and a dual-luciferase reporter assay system (Promega Corporation) was used to conduct a luciferase reporter gene assay on an LD400 luminometer (Beckman Coulter, Brea, CA, USA) according to the manufacturer's instructions. The data are presented as the ratio of Renilla luciferase to firefly luciferase.

Statistical analysis. GraphPad Prism 9 software (La Jolla, CA, USA) was used for statistical analysis. The statistical data are presented as the means \pm standard deviation (SD). Student's t-test was used to compare groups, with $p$ values $<0.05$ considered to be statistically significant.

\section{Results}

MiR-1246 suppresses the proliferation, migration, and invasion of TNBC cells. To investigate the functional role of miR-1246 in triple-negative breast cancer cells, we transfected MDA-MB-231 and BT-549 triple-negative breast cancer cell lines with a miR-1246 mimic control (miR-NC) or miR-1246 mimic. The expression levels of miR-1246 were significantly upregulated in the cells transfected with miR-1246 mimic compared with the miR-NC group after 24 hours (Fig. 1A). A CCK-8 assay showed that upregulation of miR-1246 inhibited MDA-MB-231 and BT-549 cell proliferation (Fig. 1B), and a wound healing assay showed that upregulation of miR-1246 expression also greatly inhibited the migration of MDA-MB-231 and BT-549 cells (Fig. 1C-D). In addition, we tested the effect of miR-1246 on the invasion capacity of MDA-MB-231 and BT-549 cells. As shown in Fig. 1E-F, overexpression of miR-1246 markedly 
repressed the invasive capacity of the TNBC cells. These results suggest that miR-1246 may effectively suppress the proliferation, migration and invasion of TNBC in vitro.

DYRK1A is a direct target of miR-1246 in TNBC cells. PicTar, TargetScan and miRanda were used to discover potential target genes of miR-1246. As shown in Fig. 2A, these tools revealed that there is a miR-1246-binding site in the 3'UTR of DYRK1A. Thus, mutations were introduced within the seed sequences of the 3'UTR of DYRK1A, and the wild-type and mutant 3'UTR of DYRK1A were cloned into a pmirGLO vector to obtain pmirGLO-DYRK1A-wt and pmirGLO-DYRK1A-mut plasmids. We then used a luciferase reporter gene assay to determine whether miR-1246 was able to directly bind to the core sequence of the 3'UTR of DYRK1A in the transfected 293T cells. The luciferase reporter assay showed that the luciferase activity was markedly reduced by cotransfection with miR-1246 mimic and pmirGLODYRK1A-wt plasmids, but no difference was detected in the cells cotransfected with miR-1246 mimic and pmirGLO-DYRK1A-mut (Fig. 2B). In addition, the qRT-PCR and western blotting results demonstrated that transfection with miR-1246 mimic significantly decreased DYRK1A mRNA and protein expression levels in the MDA-MB-231 cells compared to the control cells. In contrast, the miR-1246 inhibitor promoted the expression of DYRK1A (Fig. 2C-D). To confirm that miR-1246 regulated DYRK1A expression and suppressed the proliferation, migration and/or invasion of TNBC cells, miR-1246 and pcDNA3.1-MycDYRK1A plasmids were cotransfected into TNBC cells. After 48 hours, western blotting was performed to confirm DYRK1A overexpression (Fig. 3A). Significantly, the overexpression of DYRK1A reversed the proliferation, migration and invasion of MDA-MB-231 and BT-549 cells inhibited by miR-1246, as measured by CCK-8 assay, wound healing assay and Transwell invasion assay, respectively (Fig. 3B-F). These findings suggest that DYRK1A is a direct target of miR-1246 in TNBC cells and that miR-1246 may repress cell migration and invasion in TNBC cells via DYRK1A inhibition.

MiR-1246 has a suppressive role in the regulation of the EMT of TNBC cells by targeting DYRK1A. Since DYRK1A is known as a regulator of the EMT during human tumorigenesis (11) and because the current results established a relationship between miR-1246 and DYRK1A in MDA-MB-231 and BT-549 cells, we hypothesized that miR-1246 suppresses the proliferation, migration, and invasion of TNBC related to the DYRK1A-mediated EMT. Thus, we measured the expression levels of EMT-related proteins such as Ncadherin, E-cadherin, and vimentin in TNBC cells after either overexpression or knockdown of miR-1246. The western blot analysis showed that, compared with the control cells, transfection with the miR-1246 mimic increased the protein expression of E-cadherin and decreased the protein expression of $\mathrm{N}$-cadherin and vimentin in the TNBC cells. In contrast, transfection with an miR-1246 inhibitor decreased the expression of E-cadherin and increased the expression of $\mathrm{N}$-cadherin and vimentin compared to the expression in the control cells (Fig. 4A-C). Moreover, the overexpression of DYRK1A reversed the inhibition of the EMT of triple-negative breast cancer cells that had been induced by miR-1246 (Fig. 4B-D). These results indicate that miR-1246 may suppress the EMT of TNBC cells via DYRK1A function.

miR-1246/DYRK1A may suppress the EMT of TNBC cells via the Wnt/ $\beta$-catenin pathway. Since the Wnt/ $\beta$-catenin pathway is known as a regulator of the EMT during human tumorigenesis (12), we hypothesized that miR-1246/DYRK1A suppresses the EMT of TNBC cells via the Wnt/ $\beta$-catenin pathway. 
Thus, we measured the expression levels of Wnt/ $\beta$-catenin-related proteins such as $\beta$-catenin, GSK-3 $\beta$ and p-GSK-3 $\beta$ in MDA-MB-231 cells after overexpression and knockdown of miR-1246. The western blot analysis showed that, compared the control cells, transfection with the miR-1246 mimic decreased the protein expression of $\beta$-catenin and p-GSK-3 $\beta$ in the MDA-MB-231 cells. In contrast, transfection with a miR-1246 inhibitor decreased the expression of $\beta$-catenin and p-GSK-3 $\beta$ compared to that in the control cells (Fig. 5A). Furthermore, the proteins in the nucleus and cytoplasm of $\beta$-catenin were separated into fractions. The results showed a decrease in nuclear $\beta$-catenin levels by miR-1246, which was reversed by transfection with Myc-DYRK1A (Fig. 5B-C). These results indicate that miR-1246/DYRK1A may suppress the EMT of TNBC cells via the Wnt/ $\beta$-catenin pathway.

MiR-1246 suppresses the metastasis of breast cancer cells by targeting the DRAK1A/PGRN axis to prevent the epithelial-mesenchymal transition. Although elevated PGRN expression has been confirmed in breast cancer, the molecular mechanism has rarely been studied. PGRN can activate the phosphorylation of GSK3 $\beta$ to promote tumor growth $(13,14)$. PGRN has no phosphorylation function, and the relationship between PGRN and the EMT has not yet been reported. Interestingly, as shown in Fig. 6A, we found, for the first time, that overexpression of pcDNA3.1-Flag-PGRN promotes the EMT. Hence, we hypothesized that the mechanism by which PGRN can activate the phosphorylation of GSK3 $\beta$ may be related to DYRK1A because related studies have shown the phosphorylation and inactivation of GSK3 $\beta$ through DYRK1A. PGRN may interact with DYRK1A to regulate the phosphorylation of GSK3 $\beta$. Therefore, the present study examined interactions between PGRN and DYRK1A. Immunoprecipitation demonstrated that PGRN endogenously interacted with DYRK1A (Fig. 6B). To investigate the effects of the DRAK1A/PGRN axis on the inhibition of the EMT of breast cancer cells mediated by miR-1246, EMTrelated proteins such as $\mathrm{N}$-cadherin, E-cadherin, vimentin, GSK3 $\beta$ and $\mathrm{p}$-GSK3 $\beta$ were examined in the present study. The results showed that the overexpression of PGRN can promote the phosphorylation of GSK by DYRK1A and promote the occurrence of EMT in triple-negative breast cancer, but it this phosphorylation and promotion was suppressed by the upregulation of miR-1246 expression (Fig. 6C). These results indicate that miR-1246 suppresses the metastasis of breast cancer cells by targeting the DRAK1A/PGRN axis and preventing the epithelial-mesenchymal transition.

\section{Discussion}

Breast cancer is one of the most common malignant tumors that threatens the health of women around the world, and it is also one of the main malignant tumors that causes female death (1). In developing countries, breast cancer has surpassed cervical cancer as the leading cause of cancer death in women. The death toll of triple-negative breast cancer accounts for $80 \%$ of all breast cancer deaths, and due to the lack of hormone receptors, including human epidermal growth factor receptor, hormone therapy and targeted therapy elicit little response. Therefore, the 5-year survival rate of patients with TNBC is much lower than that of patients with other types of breast cancer (2). In addition, TNBC is more likely to occur in young female patients and is highly metastatic. It can relapse within 3 years and metastasize to the brain, liver, and lungs (15). To detect the metastasis of TNBC at an early stage and improve the prognosis and survival rate of patients, researchers need to gain greater knowledge and understanding of 
metastasis-related signaling pathways and biomarkers. MicroRNAs (miRNAs) are endogenous noncoding RNAs that have been confirmed by numerous studies to participate in biological processes such as cell differentiation, proliferation, metabolism and apoptosis $(6,16,17)$. Similarly, in the process of cancer occurrence and development, the expression of miRNA has been inextricably linked with the development of the disease, including the metastasis of breast cancer (18).

Tumor metastasis is a process in which malignant tumor cells detach from the primary tumor and reach secondary tissues or organs through lymphatic, blood, and other transfer methods to form tumors. It is a multistep, multistage, continuous and complex biological process involving multiple factors and multilevel regulation, including the expression of tumor metastasis-promoting and metastasissuppressing genes, the actions of cell adhesion factors and protein-degrading enzymes, and immune status(19-22). The metastasis of malignant tumors is often the main reason for the failure of tumor treatment, which leads to the death of patients. With the deepening of miRNA research, an increasing number of studies have shown that miRNAs can regulate the metastasis of malignant tumors through the EMT, cancer stem cell (CSC), tumor angiogenesis, tumor-associated macrophage (TAM) and other pathways $(6,23-25)$. TNBC is a malignant tumor derived from breast epithelial cells, and the EMT enables tumor cells derived from epithelial cells to acquire high invasive and metastatic abilities, leading to tumor invasion and metastasis. In the process of EMT induction, a variety of signaling pathways (such as the Wnt/ $\beta$-catenin, TGF- $\beta$, Notch, and PI3K signaling pathways) can eventually induce the expression transcriptional regulators such as SNAIL1, SNAIL2, TWIST, ZEB1, and ZEB2 $(26,27)$. Then, the expression of mesenchymal markers (such as vimentin, $\mathrm{N}$-cadherin, etc.) is upregulated, and the expression of epithelial markers (such as E-cadherin and zonula occluden-1) is downregulated (27). Many studies have shown that miRNAs can play a role in the EMT process by participating in the regulation of signaling pathways or act as transcriptional regulators $(6,28)$. For example, some recent studies have shown that miR-212-5p can induce the acquisition of an EMT phenotype by downregulating Prrx2 to inhibit TNBC proliferation, thereby inhibiting cell migration and invasion during tumor progression (28). In this study, we found that miR-1246 attenuated TNBC cell invasion and the EMT by targeting the DYRK1-mediated Wnt/ $\beta$-catenin pathway.

miR-1246 is a newly discovered miRNA, and research on it is in its infancy. Lin et al. (29) found that miR1246 targets CCNG2 to enhance cancer stemness and chemoresistance in oral carcinomas. Xu et al. (30) showed that miR-1246 inhibits cell invasion and the epithelial mesenchymal transition process by targeting CXCR4 in lung cancer cells. However, compared with normal breast epithelial cells, malignant breast epithelial cells are more likely to release miR-1246 into the intercellular space, milk or blood, which may reduce the degree of cell maturation that can be detected. The significant increase in the level of miR-1246 previously found in breast duct irrigation fluid indicates that atypia cells may be present (31). In this study, we explored the functional role of miR-1246 in triple-negative breast cancer cells and confirmed that DYRK1A is a direct target of miR-1246. Among the possible targets, DYRK1A has been shown by many studies to act as a proto-oncogene by promoting cell proliferation and inhibiting cell apoptosis (32). Some studies have found that DYRK1A is highly expressed in glioblastoma (GBM), and its role is related to EGFR. The inhibition of DYRK1A in early GBM cells and neural progenitor cells 
promotes the degradation of EGFR and significantly reduces the self-renewal ability of normal and tumorigenic cells. Inhibition of DYRK1A expression renders EGFR unstable and reduces the growth of GBM dependent on EGFR (33). Studies have also shown that DYRK1A inactivates nuclear factor of activated T cells (NFATC) through phosphorylation, which can improve the migration ability of breast cancer cells and the drug resistance of leukemia cells(34-35).

In recent years, studies have found that an abnormal EMT is closely related to the occurrence and development of tumors. The results of many studies have shown that multiple intracellular signal transduction pathways are involved in the EMT process, and the most extensively studied is the Wnt/ $\beta$ catenin signaling pathway $(3,5)$. GSK-3 $\beta$ and $\beta$-catenin are both key regulators of Wnt/ $\beta$-catenin signaling (3-4). Since miR-1246 reduces the migration, invasion and EMT of breast cancer cells (Fig. 2), we further investigated whether miR-1246 affects the expression of GSK-3 $\beta$ and $\beta$-catenin during breast cancer cell metastasis. The results of overexpression, knockout and supplementary experiments showed

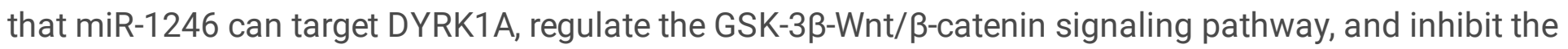
EMT of breast cancer cells. In recent years, studies have found that PGRN plays important roles in the proliferation, drug resistance, migration, and invasion of breast cancer, ovarian cancer, liver cancer and other tumors $(13,14,36)$. In addition, serum PGRN levels are related to the treatment, survival and progression of breast cancer. A previous study has revealed significant differences in serum PGRN levels between healthy subjects without a history of breast cancer and patients with breast cancer at various stages; notably, the higher the serum PGRN content was, the worse the clinical treatment of the breast cancer patients (37). This evidence indicates that PGRN may become a novel target for the clinical treatment of breast cancer, but the related molecular mechanisms have been rarely reported. In this study, PGRN regulated the phosphorylation of GSK-3 $\beta$ to promote the EMT of breast cancer cells (Fig. 6). PGRN has been previously shown incapable of activating substrate phosphorylation, suggesting that PGRN played a cofactor function in regulating GSK-3 $\beta$ phosphorylation in this study. According to related reports, phosphorylation and inactivation of gGSK-3 $\beta$ occur by dual-specificity tyrosine phosphorylationregulated kinase 1A (Dyrk1A). In this study, we confirmed that PGRN can interact with DYRK1A and may facilitate the GSK-3 $\beta$ phosphorylation process, and the DRAK1A/PGRN axis that is formed prevents breast cancer cells from undergoing the EMT through the regulatory action of miR-1246.

\section{Conclusion}

In the present study, we examined the effects of miR-1246 on the proliferation, invasion, and metastasis of TNBC cells and confirmed that DYRK1A is a direct target of miR-1246 and that miR-1246 suppresses the metastasis of breast cancer cells by inactivating their epithelial-mesenchymal transition by targeting the DRAK1A/PGRN axis. The present data provide novel insights into the mechanism critical to the progression of human TNBC. Taken together, these data suggest that miR-1246 may be a novel target for the treatment of triple-negative breast cancer.

\section{Declarations}


Acknowledgements

None

\section{Authors' contributions}

Yichao Wang conceived the study. Pan Wang searched the literature and collected the data. Pan Wang, Wenju Chen, Yaqiong Zhang, Zhaoyun Li, Qianyi Zhong performed the statistical analysis. Pan Wang drafted the manuscript. Yichao Wang reviewed the manuscript. All authors have read and approved the final paper.

\section{Funding}

The project were supported by Zhejiang Science and Technology of Analysis and Testing Planning Project (grant no.2018C37037), and Taizhou Science and Technology Plan Project (grant no.1901ky35).

\section{Availability of data and materials}

All data generated or analyzed during this study are included within the article.

\section{Declarations}

Ethics approval and consent to participate

Written informed consents were obtained from all participants, and this study was permitted by the Ethics Committee of Taizhou Central Hospital

Consent for publication

Not applicable.

Competing interests

The authors declare that they have no conflict of interest.

\section{Footnotes}

\section{Publisher's Note}

Springer Nature remains neutral with regard to jurisdictional claims in published maps and institutional affiliations.

\section{References}

1. Ferlay J, Colombet M, Soerjomataram I, Parkin DM, Piñeros M, Znaor A, Bray F. Cancer statistics for the year 2020: an overview. Int J Cancer. 2021. 
2. Foulkes WD, Smith IE, Reis-Filho JS. Triple-negative breast cancer. N Engl J Med. 2010;363(20):1938-48.

3. Khaled N, Bidet Y. New Insights into the Implication of Epigenetic Alterations in the EMT of Triple Negative Breast Cancer. Cancers (Basel). 2019;11(4):559.

4. Pastushenko I, Blanpain C. EMT Transition States during Tumor Progression and Metastasis. Trends Cell Biol. 2019;29(3):212-26.

5. Yilmaz M, Christofori G. EMT, the cytoskeleton, and cancer cell invasion. Cancer Metastasis Rev. 2009;28(1-2):15-33.

6. Nieszporek A, Skrzypek K, Adamek G, Majka M. Molecular mechanisms of epithelial to mesenchymal transition in tumor metastasis. Acta Biochim Pol. 2019;66(4):509-20.

7. Wang Z, Li Y, Ahmad A, Azmi AS, Kong D, Banerjee S, Sarkar FH. Targeting miRNAs involved in cancer stem cell and EMT regulation: An emerging concept in overcoming drug resistance. Drug Resist Updat. 2010;13(4-5):109-18.

8. Lu J, Getz G, Miska EA, Alvarez-Saavedra E, Lamb J, Peck D, Sweet-Cordero A, Ebert BL, Mak RH, Ferrando AA, Downing JR, Jacks T, Horvitz HR, Golub TR. MicroRNA expression profiles classify human cancers. Nature. 2005;435(7043):834-8.

9. Liu HT, Fan WX. MiRNA-1246 suppresses the proliferation and migration of renal cell carcinoma through targeting CXCR4. Eur Rev Med Pharmacol Sci. 2020;24(11):5979-87.

10. Cheng Y, Li Z, Xie J, Wang P, Zhu J, Li Y, Wang Y. MiRNA-224-5p inhibits autophagy in breast cancer cells via targeting Smad4. Biochem Biophys Res Commun. 2018;506(4):793-8.

11. Ding D, Li C, Zhao T, Li D, Yang L, Zhang B. LncRNA H19/miR-29b-3p/PGRN Axis Promoted EpithelialMesenchymal Transition of Colorectal Cancer Cells by Acting on Wnt Signaling. Mol Cells. 2018;41(5):423-35.

12. Li J, Zhou BP. Activation of $\beta$-catenin and Akt pathways by Twist are critical for the maintenance of EMT associated cancer stem cell-like characters. BMC Cancer. 2011;11:49.

13. Gao X, Joselin AP, Wang L, Kar A, Ray P, Bateman A, Goate AM, Wu JY. Progranulin promotes neurite outgrowth and neuronal differentiation by regulating GSK-3ß. Protein Cell. 2010;1(6):552-62.

14. Nedachi T, Kawai T, Matsuwaki T, Yamanouchi K, Nishihara M. Progranulin enhances neural progenitor cell proliferation through glycogen synthase kinase $3 \beta$ phosphorylation. Neuroscience. 2011;185:106-15.

15. O'Reilly D, Sendi MA, Kelly CM. Overview of recent advances in metastatic triple negative breast cancer. World J Clin Oncol. 2021;12(3):164-82.

16. Tay Y, Zhang J, Thomson AM, Lim B, Rigoutsos I. MicroRNAs to Nanog, Oct4 and Sox2 coding regions modulate embryonic stem cell differentiation. Nature. 2008;455(7216):1124-8.

17. Chang TC, Wentzel EA, Kent OA, Ramachandran K, Mullendore M, Lee KH, Feldmann G, Yamakuchi M, Ferlito M, Lowenstein CJ, Arking DE, Beer MA, Maitra A, Mendell JT. Transactivation of miR-34a by p53 broadly influences gene expression and promotes apoptosis. Mol Cell. 2007;26(5):745-52. 
18. Ma L, Teruya-Feldstein J, Weinberg RA. Tumour invasion and metastasis initiated by microRNA-10b in breast cancer. Nature. 2007;449(7163):682-8.

19. Albini A, Mirisola V, Pfeffer U. Metastasis signatures: genes regulating tumor-microenvironment interactions predict metastatic behavior. Cancer Metastasis Rev. 2008;27(1):75-83.

20. Yan J, Yang Q, Huang Q. Metastasis suppressor genes. Histol Histopathol. 2013;28(3):285-92.

21. Kim KS, Kim J, Oh N, Kim MY, Park KS. ELK3-GATA3 axis modulates MDA-MB-231 metastasis by regulating cell-cell adhesion-related genes. Biochem Biophys Res Commun. 2018;498(3):509-15.

22. Wrobel P, Ahmed S. Current status of immunotherapy in metastatic colorectal cancer. Int J Colorectal Dis. 2019;34(1):13-25.

23. Chakraborty C, Chin KY, Das S. miRNA-regulated cancer stem cells: understanding the property and the role of miRNA in carcinogenesis. Tumour Biol. 2016;37(10):13039-48.

24. Goradel NH, Mohammadi N, Haghi-Aminjan H, Farhood B, Negahdari B, Sahebkar A. Regulation of tumor angiogenesis by microRNAs: State of the art. J Cell Physiol. 2019;234(2):1099-110.

25. Chatterjee B, Saha P, Bose S, Shukla D, Chatterjee N, Kumar S, Tripathi PP, Srivastava AK. MicroRNAs: As Critical Regulators of Tumor- Associated Macrophages. Int J Mol Sci. 2020;21(19):7117.

26. Gonzalez DM, Medici D. Signaling mechanisms of the epithelial-mesenchymal transition. Sci Signal. 2014;7(344):re8.

27. Khaled N, Bidet Y. New Insights into the Implication of Epigenetic Alterations in the EMT of Triple Negative Breast Cancer. Cancers (Basel). 2019;11(4):559.

28. Lv ZD, Yang DX, Liu XP, Jin LY, Wang XG, Yang ZC, Liu D, Zhao JJ, Kong B, Li FN, Wang HB. MiR-212$5 p$ Suppresses the Epithelial-Mesenchymal Transition in Triple-Negative Breast Cancer by Targeting Prrx2. Cell Physiol Biochem. 2017;44(5):1785-95.

29. Lin SS, Peng CY, Liao YW, Chou MY, Hsieh PL, Yu CC. miR-1246 Targets CCNG2 to Enhance Cancer Stemness and Chemoresistance in Oral Carcinomas. Cancers (Basel). 2018;10(8):272.

30. Xu X, Cao L, Zhang Y, Lian H, Sun Z, Cui Y. MicroRNA-1246 inhibits cell invasion and epithelial mesenchymal transition process by targeting CXCR4 in lung cancer cells. Cancer Biomark. 2018;21(2):251-60.

31. Zhai LY, Li MX, Pan WL, Chen Y, Li MM, Pang JX, Zheng L, Chen JX, Duan WJ. In Situ Detection of Plasma Exosomal MicroRNA-1246 for Breast Cancer Diagnostics by a Au Nanoflare Probe. ACS Appl Mater Interfaces. 2018;10(46):39478-86.

32. Laham AJ, Saber-Ayad M, El-Awady R. DYRK1A: a down syndrome-related dual protein kinase with a versatile role in tumorigenesis. Cell Mol Life Sci. 2021;78(2):603-19.

33. Pozo N, Zahonero C, Fernández P, Liñares JM, Ayuso A, Hagiwara M, Pérez A, Ricoy JR, HernándezLaín A, Sepúlveda JM. Sánchez-Gómez P. Inhibition of DYRK1A destabilizes EGFR and reduces EGFR-dependent glioblastoma growth. J Clin Invest. 2013;123(6):2475-87.

34. Stefos GC, Soppa U, Dierssen M, Becker W. NGF upregulates the plasminogen activation inhibitor-1 in neurons via the calcineurin/NFAT pathway and the Down syndrome-related proteins DYRK1A and 
RCAN1 attenuate this effect. PLoS One. 2013;8(6):e67470.

35. Malinge S, Bliss-Moreau M, Kirsammer G, Diebold L, Chlon T, Gurbuxani S, Crispino JD. Increased dosage of the chromosome 21 ortholog Dyrk1a promotes megakaryoblastic leukemia in a murine model of Down syndrome. J Clin Invest. 2012;122(3):948-62.

36. Liu C, Li J, Shi W, Zhang L, Liu S, Lian Y, Liang S, Wang H. Progranulin Regulates Inflammation and Tumor. Antiinflamm Antiallergy Agents Med Chem. 2020;19(2):88-102.

37. Koo DH, Park CY, Lee ES, Ro J, Oh SW. Progranulin as a prognostic biomarker for breast cancer recurrence in patients who had hormone receptor-positive tumors: a cohort study. PLoS One. 2012;7(6):e39880.

\section{Figures}

A.

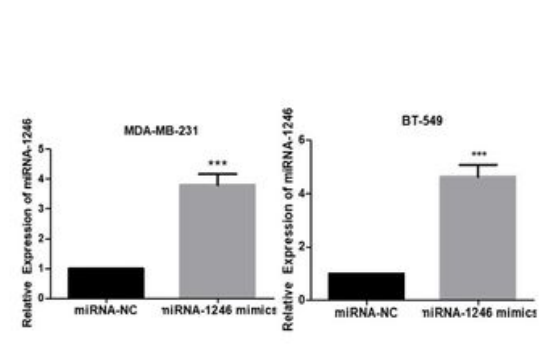

B.

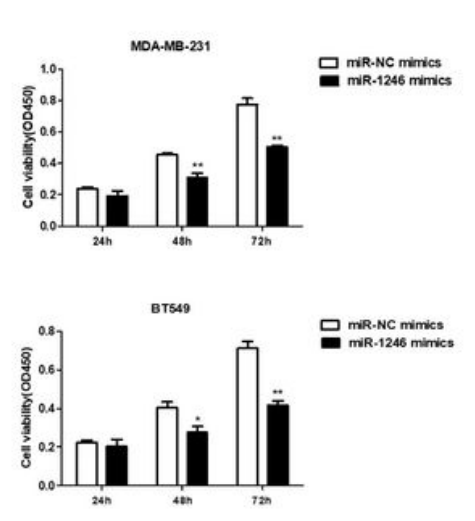

C.

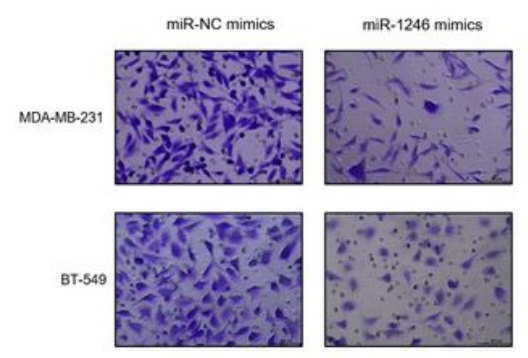

MDA.MB.23T
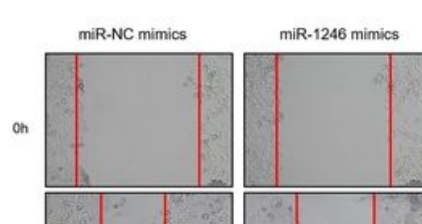

Bт.549

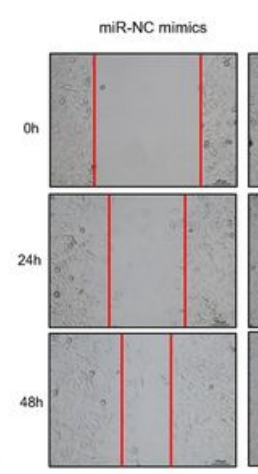

mir-1246 mimics

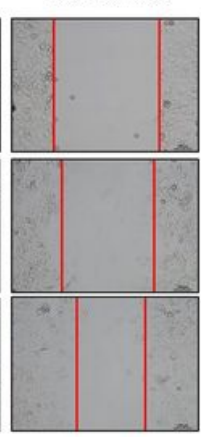

D.

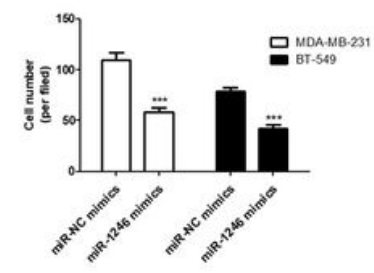

F.

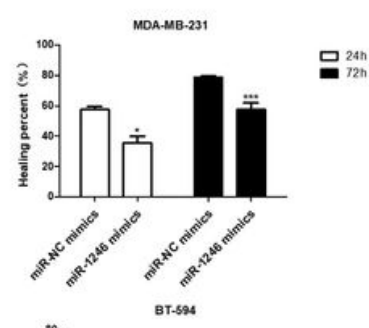

믐 ${ }^{24 \mathrm{~h}}$

\section{Figure 1}

miR 1246 suppresses the proliferation, migration, and invasion of TNBC cells. (A) RT-qPCR was performed to assess the relative miR 1246 levels in MDA-MB-231 and BT-549 cells after transfection with miR-1246 mimics or miR-NC. (B) CCK-8 assay, (C) Transwell assay and (D) wound healing assay were performed to observe the proliferation, migration, and invasive capacity of TNBC cells after transfection with miR-NC mimic or miR 1246 mimic. ${ }^{*} p<0.05,{ }^{* *} p<0.01,{ }^{* \star *} p<0.001$ vs. miR-NC. 
A.

miR-1246 3' ggacgAGGUUUUUAGGUAa 5' DYRK1A 3'UTR $\quad 5^{\prime}$........UCAGGAAAUCCAUa $3^{\prime}$

C.

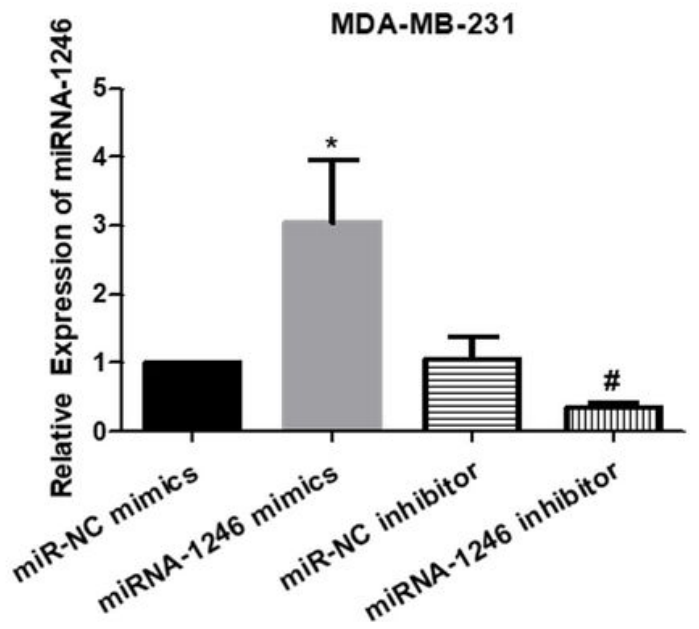

B.

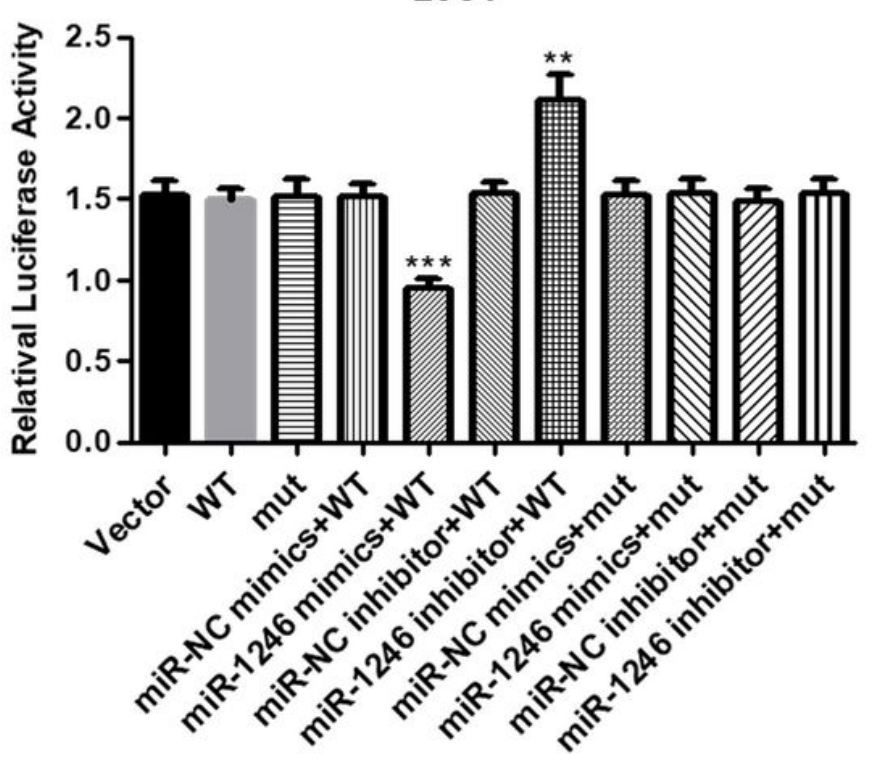

MDA-MB-231

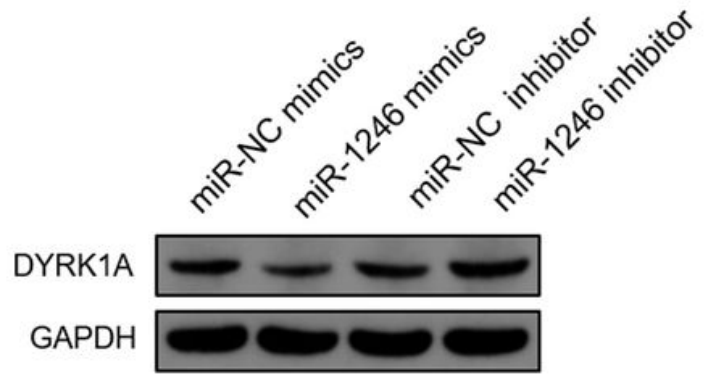

Figure 2

DYRK1A is a direct target of miR-1246 in TNBC cells. (A) Predicted human DYRK1A 3'UTR binding site for miR-1246. (B) Relative luciferase activity was assessed in HEK293T cells cotransfected with pmirGLODYRK1A-wt or pmirGLO-DYRK1A-mut plasmid, miR-1246 mimic or miR-NC mimic, and an miR-1246 inhibitor or miR-NC mimic. (C) Relative miR-1246 levels in MDA-MB-231 cells after transfection with miR1246 mimic or miR-NC mimic and an miR-1246 inhibitor or miR-NC inhibitor, as determined by RT-qPCR.

(D) Protein expression in MDA-MB-231 cells following transfection with miR-1246 mimic or miR-NC mimic and an miR-1246 inhibitor or miR-NC inhibitor, as determined by western blotting using GAPDH as an internal reference. ${ }^{*} p<0.05,{ }^{*} p<0.01,{ }^{* \star *} p<0.001$ vs. control cells. 


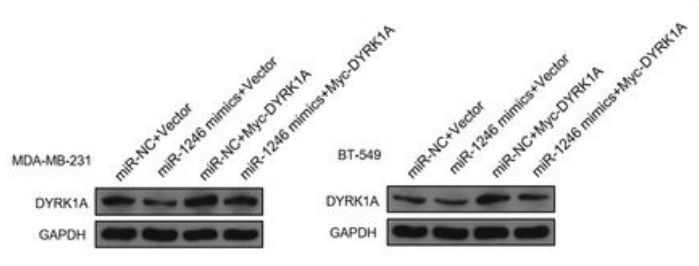

B.
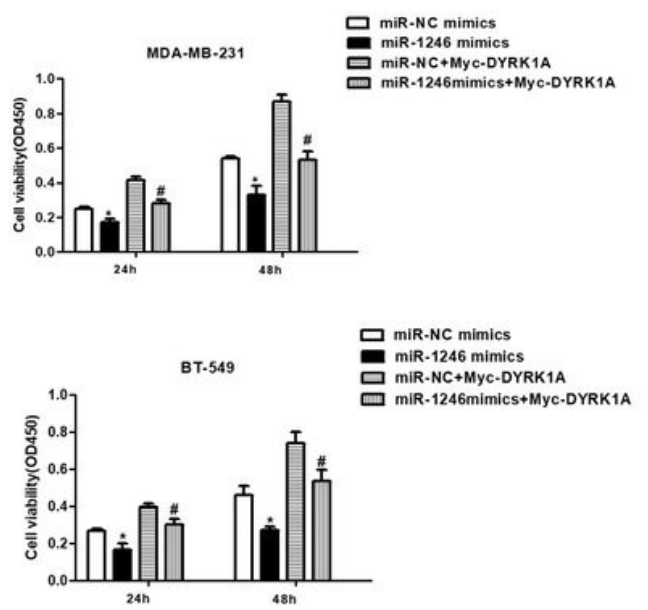

C.
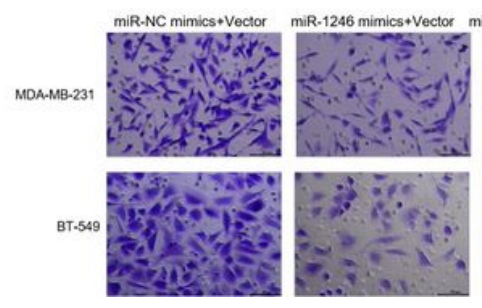

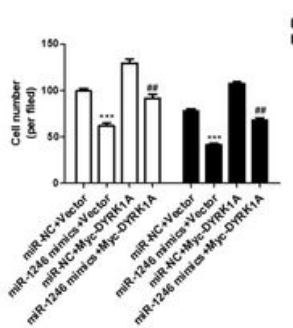

E.
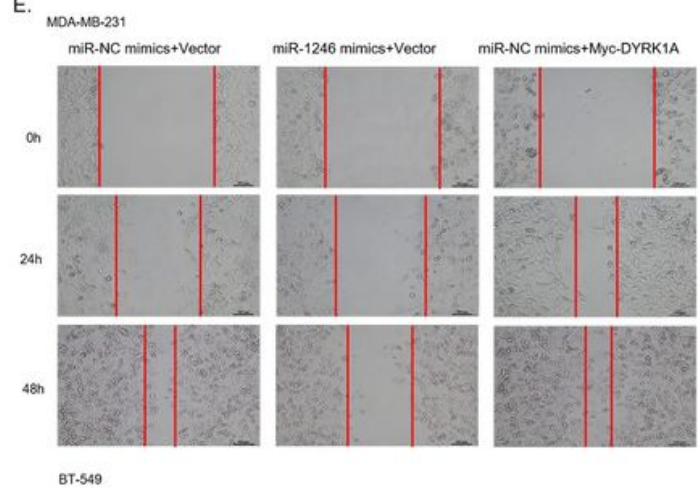

miR-NC mimics+vector
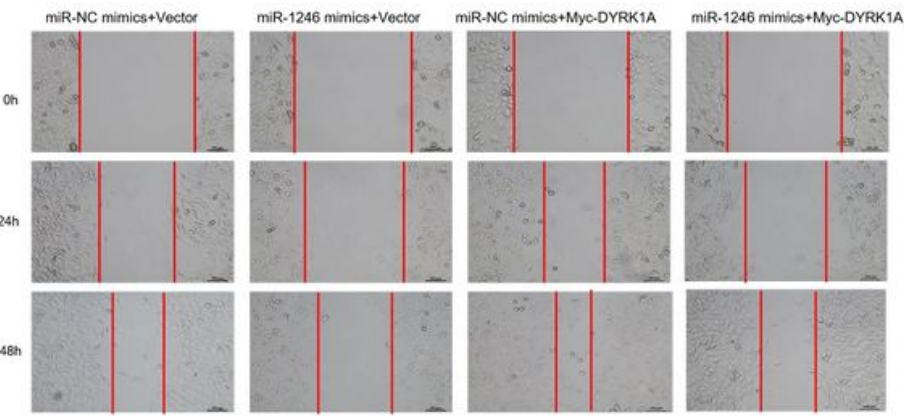

Figure 3

The overexpression of DYRK1A reversed the proliferation, migration and invasion of TNBC cells inhibited by miR-1246. (A) The expression of DYRK1A in MDA-MB-231 and BT-549 cells after transfection with Myc-DYRK1A and miR-1246 mimic or miR-NC mimic was assessed by western blotting. (B) CCK-8 assay, (C) Transwell assay and (D) wound healing assay were used to observe the proliferation, migration, and invasive capacity of TNBC cells after transfection with Myc-DYRK1A and miR-NC mimic or miR-1246 mimic. ${ }^{*} p<0.05,{ }^{* \star} p<0.01,{ }^{* \star *} p<0.001$ vs. miR-NC mimic; $\# p<0.05, \# \# p<0.01, \# \# \# p<0.001$ vs. miR-1246 mimic. 
A.

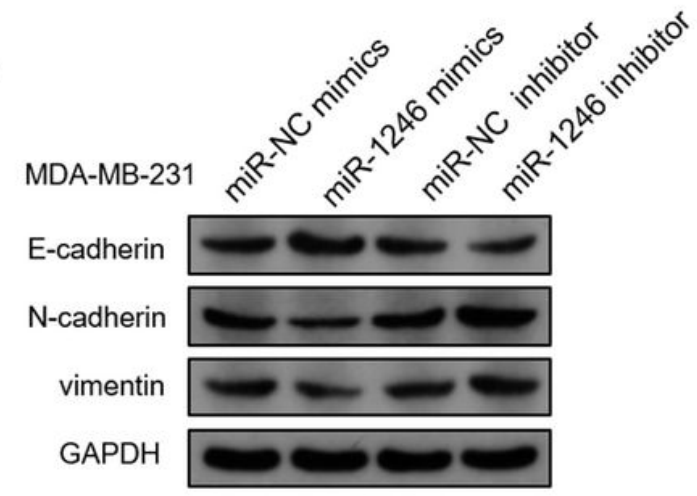

C.

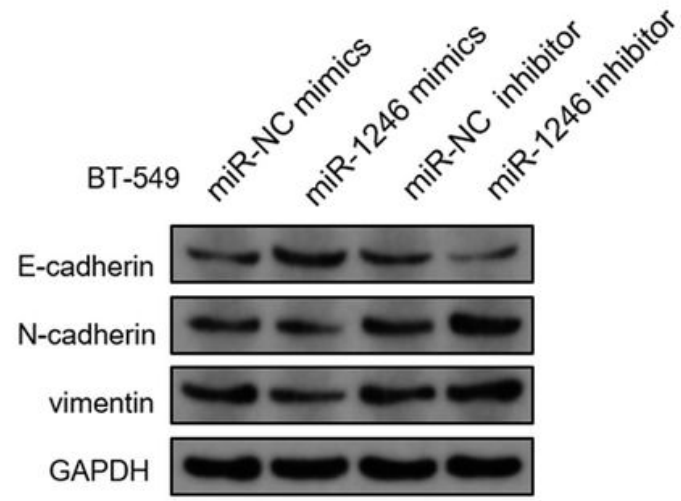

B.

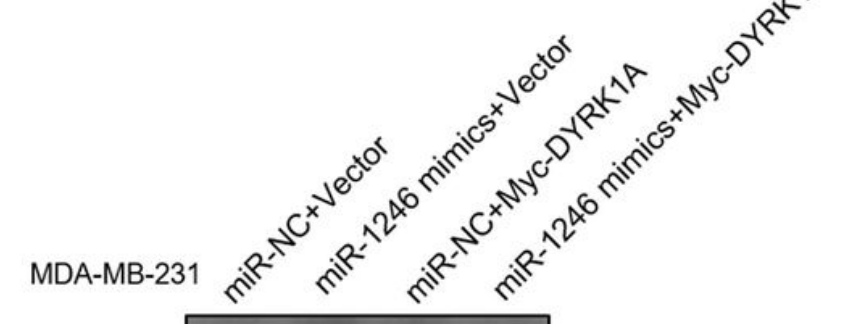

D.

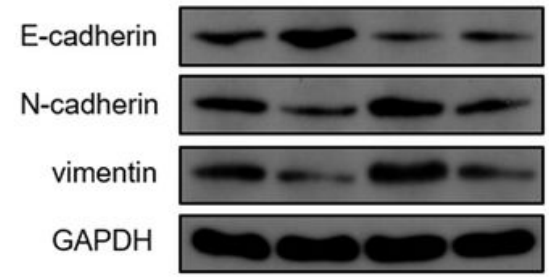

BT-549

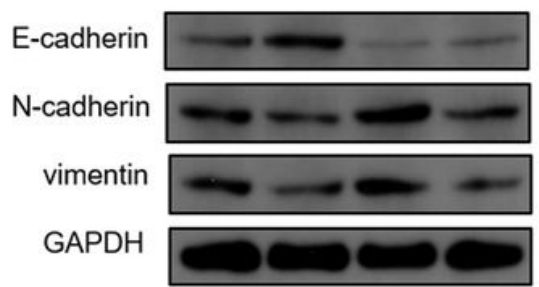

\section{Figure 4}

miR-1246 plays a suppressive role in the regulation of the EMT of TNBC cells by targeting DYRK1A. (A-C) The expression of EMT-related proteins was assessed in MDA-MB-231 and BT-549 cells after transfection with miR-1246 mimic or miR-NC mimic and an miR-1246 inhibitor or miR-NC inhibitor by western blotting. (B-D) The overexpression of DYRK1A activated the EMT of TNBC cells that had been inhibited by miR1246.

A.

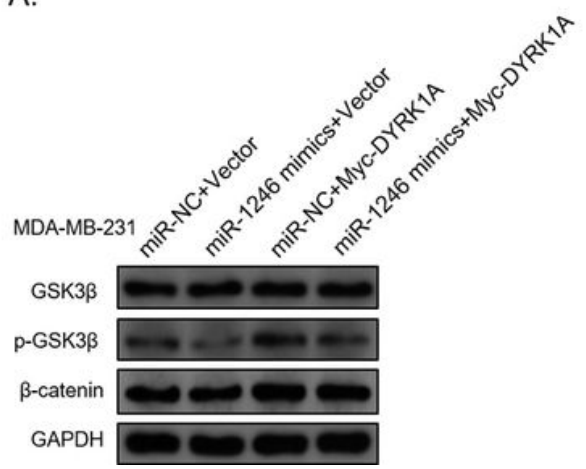

B.

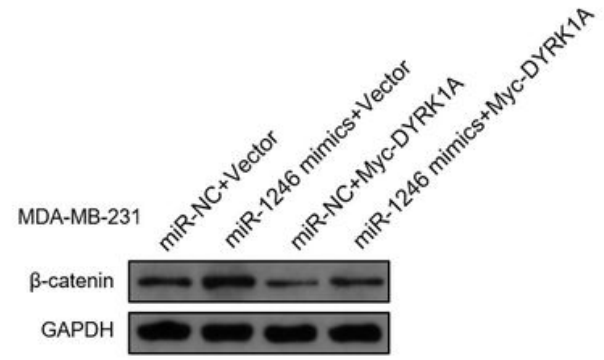

C.

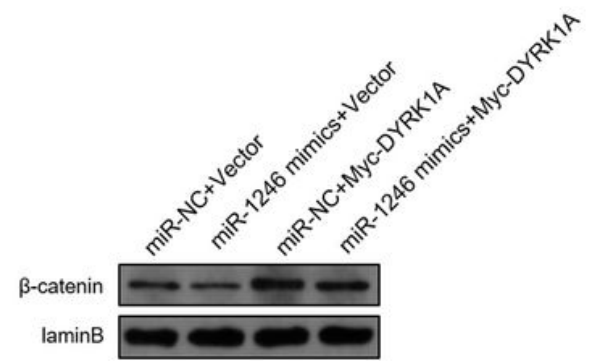

Figure 5 
miR-1246/DYRK1A may suppress the EMT of TNBC cells via the Wnt/ $\beta$-catenin pathway. (A) The expression of GSK3 $\beta$, p-GSK3 $\beta$, and $\beta$-catenin was assessed in MDA-MB-231 cells after transfection with miR-1246 mimic or miR-NC mimic and Myc-DYRK1A by western blotting. (B-C) The separation of $\beta$ catenin proteins into nuclear and cytoplasmic fractions showed decreased nuclear translocation of $\beta$ catenin in the presence of miR-1246, which was reversed upon transfection with Myc-DYRK1A. GAPDH was used as an internal reference for measuring cytoplasmic protein levels, and lamin B was used as the normalization control for measuring the nuclear protein levels.

A.

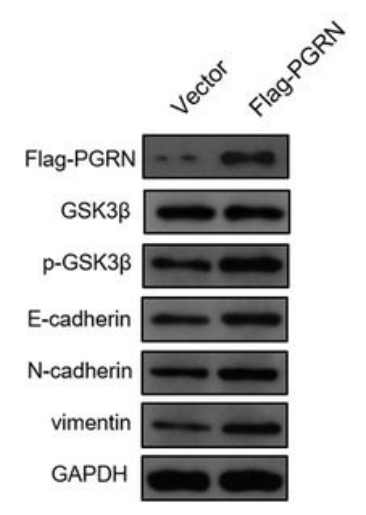

B.

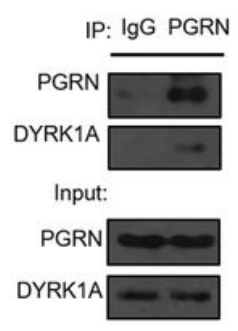

C.

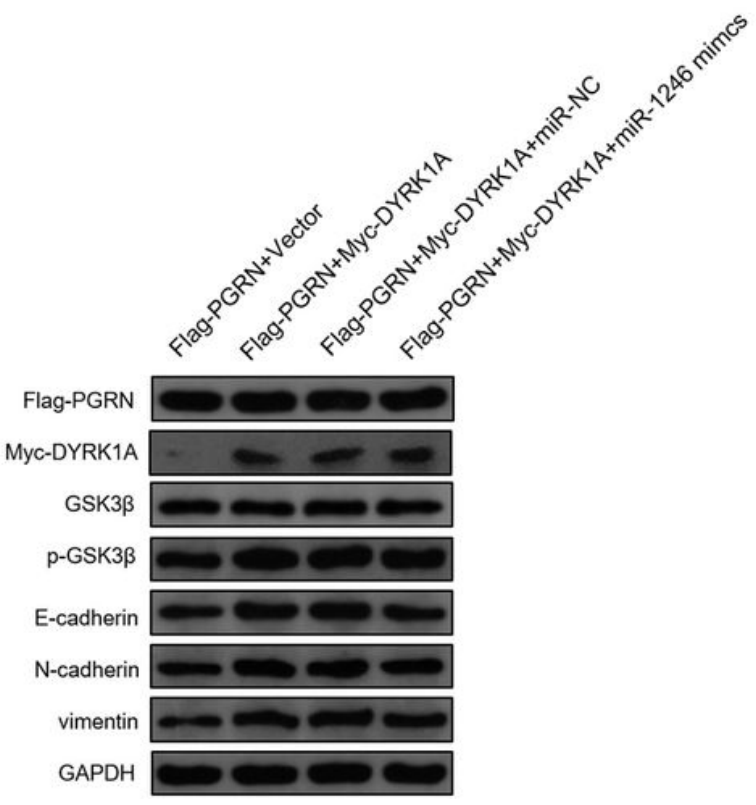

\section{Figure 6}

MiR-1246 suppresses the metastasis of breast cancer cells by targeting the DRAK1A/PGRN axis and preventing the epithelial-mesenchymal transition. (A) The expression of GSK3 $\beta$, p-GSK3 $\beta$ and EMT-related proteins was assessed in MDA-MB-231 cells after transfection with Flag-PGRN or vector by western blotting. (B) Endogenous PGRN associates with DYRK1A. MDA-MB-231 whole-cell lysates were subjected to immunoprecipitation with an anti-PGRN antibody and subsequently probed with an anti-PGRN or antiDYRK1A antibody. (C) The expression of GSK3 $\beta$, p-GSK3 $\beta$ and EMT-related proteins was assessed in MDA-MB-231 cells after transfection with Flag-PGRN or Myc-DYRK1A and miR-1246 mimic by western blotting. 